Proc. Indian Acad. Sci. (Math. Sci.), Vol. 103, No. 3, December 1993, pp. 329-332.

(C) Printed in India.

\title{
On infinitesimal $\sigma$-fields generated by random processes
}

\author{
VIVEK S BORKAR \\ Department of Electrical Engineering, Indian Institute of Science, Bangalore 560012, India
}

MS received 18 October 1992; revised 11 June 1993

\begin{abstract}
It is proved that the infinitesimal look-ahead and look-back $\sigma$-fields of a random process disagree at atmost countably many time instants.
\end{abstract}

Keywords. Random processes; look-ahead $\sigma$-field; look-back $\sigma$-field; Markov processes.

Let $X(t), t \geqslant 0$, be a Polish space-valued random process defined on a probability space $(\Omega, \mathscr{F}, P)$, where $\mathscr{F}$ is the $P$-completion of a countably generated $\sigma$-field $\mathscr{F}_{0}$. Let $\xi(t+)$ (the 'look-ahead' $\sigma$-field at $t$ ) and $\xi(t-)$ (the 'look-back' $\sigma$-field at $t$ ) denote the $P$-completions of $\cap_{s>t} \sigma(X(y), t \leqslant y \leqslant s)$ and $n_{s<t} \sigma(X(y), s \leqslant y \leqslant t)$ respectively. The aim of this note is to prove the following fact:

Theorem 1. $\xi(t+)=\xi(t-)$ for all but at most countably many $t \geqslant 0$.

We shall prove this through a sequence of lemmas. Let $\left\{A_{n}, n \geqslant 1\right\}$ be a dense collection of sets in $\mathscr{F}_{0}$ which generates $\mathscr{F}_{0}$.

Lemma 1. The space of all P-complete sub- $\sigma$-fields of $\mathscr{F}$ forms a metric space under the metric

$$
\mathrm{d}\left(\mathscr{G}_{1}, \mathscr{G}_{2}\right)=\sum_{n} 2^{-n} E\left[\left|P\left(A_{n} / \mathscr{G}_{1}\right)-P\left(A_{n} / \mathscr{G}_{2}\right)\right|\right]
$$

The proof is easy.

Remark. This topology was first introduced in Cotter [3].

For $t \geqslant 0$, let

$$
\mathscr{F}(t)=\sigma(X(s), 0 \leqslant s \leqslant t), \quad \mathscr{F}(t+)=\bigcap_{s>t} \mathscr{F}(s), \quad \mathscr{F}(t-)=\bigvee_{s<t} \mathscr{F}(s),
$$

all completed with respect to $P$. For $n \geqslant 1, t \geqslant 0$, define

$$
h_{n}(t)=E\left[\left(E\left[I_{A_{n}} / \mathscr{F}(t)\right]\right)^{2}\right] .
$$

Lemma 2. (i) $t \rightarrow h_{n}(t)$ is bounded nondecreasing for all $n$.

(ii) $\lim _{s \downarrow \downarrow t} h_{n}(s)=E\left[\left(E\left[I_{A_{*}} / \mathscr{F}(t+)\right]\right)^{2}\right]$.

(iii) $\lim _{s \uparrow \uparrow t} h_{n}(s)=E\left[\left(E\left[I_{A_{n}} / \mathscr{F}(t-)\right]\right)^{2}\right]$. 
Proof. (i) follows from the conditional Jensen's inequality and (ii), (iii) follow from the convergence theorems for regular martingales and reversed martingales [2].

From (i) above, it follows that each $h_{n}(\cdot)$ has at most a countable set of points of discontinuity. Let $D \subset[0, \infty)$ be the at most countable set of points where one or more of the $h_{n}(\cdot)$ 's is discontinuous.

Lemma 3. For $t \notin D, \mathscr{F}(t+)=\mathscr{F}(t-)$.

Proof. For $t \notin D$, Lemma 2 (ii), (iii) imply that

Thus

$$
E\left[\left(E\left[I_{A_{n}} / \mathscr{F}(t+)\right]\right)^{2}\right]=E\left[\left(E\left[I_{A_{n}} / \mathscr{F}(t-)\right]\right)^{2}\right], \quad n \geqslant 1 .
$$

$$
E\left[\left(E\left[I_{A_{n}} / \mathscr{F}(t+)\right]-E\left[\left(E\left[I_{A_{n}} / \mathscr{F}(t-)\right]\right)^{2}\right]=0, n \geqslant 1,\right.\right.
$$

implying $E\left[I_{A_{n}} / \mathscr{F}(t+)\right]=E\left[I_{A_{n}} / \mathscr{F}(t-)\right]$ a.s., $n \geqslant 1$. The claim follows from Lemma 1.

\section{COROLLARY 1}

$\xi(t+) \subset \xi(t-)$ for all but at most countably many $t$.

Proof. Let $\left\{r_{m}\right\}$ be an enumeration of rationals in $[0, \infty)$. Define $\mathscr{F}^{m}(t), \mathscr{F}^{m}(t+)$, $\mathscr{F}^{m}(t-), h_{n}^{m}(t)$ as in (1)-(4) resp. with $X\left(r_{m}+.\right)$ replacing $X(\cdot), m \geqslant 1$. The foregoing results hold for each $X\left(r_{m}+\right.$.) as well. For every rational $r \geqslant 0$, let

$$
\begin{aligned}
& D_{r}=\left\{t>r \mid P \text {-completion of } \underset{\varepsilon>0}{\bigvee} \sigma\left(X_{s}, r \leqslant s \leqslant t-\varepsilon\right) \neq\right. \\
& \left.\quad P \text {-completion of } \bigcap_{\varepsilon>0} \sigma\left(X_{s}, r \leqslant s \leqslant i+\varepsilon\right)\right\}, \\
& \bar{D}=\cup D_{r} .
\end{aligned}
$$

Then by Lemma 3, $D_{r}$ and therefore $\bar{D}$ is at most countable. Fix $t \notin \bar{D}$ and let $\left\{r_{m(i)}\right\}$ be a collection of rationals increasing to $t$. Then for $i \geqslant 1$,

$$
\bigvee_{\varepsilon>0} \sigma\left(X_{s}, r_{m(i)} \leqslant s \leqslant t-\varepsilon\right)=\bigcap_{\varepsilon>0} \sigma\left(X_{s}, r_{m(i)} \leqslant s \leqslant t+\varepsilon\right)
$$

on P-completion. Thus

$$
\xi(t+) \subset P \text {-completion of } \underset{\varepsilon>0}{\bigvee} \sigma\left(X_{i}, r_{m(i)} \leqslant s \leqslant t-\varepsilon\right), \quad i \geqslant 1
$$

and hence

$$
\xi(t+) \subset P \text {-completion of } \bigcap_{i} \underset{\varepsilon>0}{\bigvee} \sigma\left(X_{s}, r_{m(i)} \leqslant s \leqslant t-\varepsilon\right) \subset \xi(t-)
$$


The claim follows.

Proof of Theorem 1. It suffices to consider $t \in[0, T]$ for some finite $T>0$. Applying the above corollary to the process $X(T-t), t \in[0, T]$, we conclude that $\xi(t-) \subset \xi(t+)$ except at most countably many $t$. Combine this with the corollary to conclude.

\section{COROLLARY 2}

If $X(\cdot)$ is a Markov process, then $\xi(t+)=\xi(t-)=\xi(t)(\triangleq$ the P-completion of $\sigma(X(t)))$, at all but at most countably many $t$.

Proof. Note that $\xi(t) \subset \xi(t+) \cap \xi(t-)$ for all $t \geqslant 0$. Let $t \geqslant 0$ be such that $\xi(t+)=\xi(t-)$. Since $X(\cdot)$ is Markov, $\xi(t+), \xi(t-)$ are conditionally independent given $X(t)$. Thus $\xi(t+)$ is conditionally independent of itself given $X(t)$, implying $\xi(t+) \subset \xi(t)$. Similarly $\xi(t-) \subset \xi(t)$.

It is conjectured that the conclusions of Corollary 2 hold even in absence of the Markov property. If true, this result will have important implications in stochastic control theory [1]. We conclude with an example to show that one cannot improve on Theorem 1 in general.

Example Let $\Omega=[0,1]^{\infty}, \mathscr{F}_{0}=$ the product Borel $\sigma$-field, $P=$ the product Lebesgue measure and $\mathscr{F}$ the product $\sigma$-field completed with respect to $P$. Let $w=\left(w_{1}, w_{2}, \cdots\right)$ denote a typical element of $\Omega$. Let $\left\{r_{n}\right\}$ be an enumeration of rationals in $(0,1)$. Define an $R^{\infty}$-valued process $X(i)=\left[X_{1}(t), X_{2}(t), \cdots\right], t \in[0,1]$ as follows: $\left[X_{1}(t), X_{2}(t), \cdots\right]$ evaluated at the sample point $\left[w_{1}, w_{2}, \cdots\right]$ is given by

$$
\begin{aligned}
& X_{2 i}(t)=w_{2 i}\left[\left(t-r_{i}\right)^{+}\right], i \geqslant 1, \\
& X_{2 i-1}(t)=w_{2 i-1}\left[\left(t-r_{i}\right)^{-}\right], \quad i \geqslant 1,
\end{aligned}
$$

for $t \in[0,1]$. Then it is easy to see that $\xi(t+), \xi(t-), \xi(t)$ are the $P$-completions of $\Pi_{n} G_{n}^{+}(t), \Pi_{n} G_{n}^{-}(t), \Pi_{n} G_{n}^{0}(t)$ respectively, where $G_{n}^{+}(t), G_{n}^{-}(t), G_{n}^{0}(t)$ are as described below: Let $\mathfrak{B}=$ the Borel $\sigma$-field of $[0,1], \beta=$ the trivial $\sigma$-field $\{\phi,[0,1]\}$ on $[0,1]$. Then for $n \geqslant 1$,

$$
\begin{aligned}
G_{2 n}^{+}(t) & =\mathfrak{B} \text { if } t \geqslant r_{n},=\beta \text { otherwise, } \\
G_{2 n-1}^{+}(t) & =\mathfrak{B} \text { if } t<r_{n},=\beta \text { otherwise, } \\
G_{2 n}^{-}(t) & =\mathfrak{B} \text { if } t>r_{n},=\beta \text { otherwise, } \\
G_{2 n-1}^{-}(t) & =\mathfrak{B} \text { if } t \leqslant r_{n},=\beta \text { otherwise, } \\
G_{2 n}^{0}(t) & =\mathfrak{B} \text { if } t>r_{n},=\beta \text { otherwise, } \\
G_{2 n-1}^{0}(t) & =\mathfrak{B} \text { if } t<r_{n},=\beta \text { otherwise. }
\end{aligned}
$$

It follows that $\xi(t+)=\xi(t-)=\xi(t)$ for $t$ irrational in $[0,1]$ whereas $\xi(t+) \neq \xi(t-) \neq$ $\xi(t)$ for $t \in\left\{r_{n}, n \geqslant 1\right\}$. 
Remark: One may replace $X(\cdot)$ above by a real valued process without altering the conclusions by virtue of the isomorphism theorem for Polish spaces (Theorem 2.12, p. 14, of [4]).

\section{Acknowledgements}

This work is supported by grant No. 26/01/92-G from the Department of Atomic Energy, Government of India.

\section{References}

[1] Borkar V S, On extremal solutions to stochastic control problems II. Appl. Math. Optim. 28(1993) 49-56

[2] Chow Y S and Teicher H, Probability theory - independence, interchangeability, martingales (New York: Springer Verlag) (1978)

[3] Cotter K D, Similarity of information and behaviour with a pointwise convergence topology, J. Math. Econ. 15 (1986) 25-38

[4] Parthasarathy K R, Probability measures on metric spaces (New York: Academic Press) (1967) 